\title{
Dual Cholinergic Control of Fast-Spiking Interneurons in the Neostriatum
}

\author{
Tibor Koós and James M. Tepper \\ Center for Molecular and Behavioral Neuroscience, Rutgers, The State University of New Jersey, Newark, \\ New Jersey 07102
}

\begin{abstract}
GABAergic interneurons appear to play a fundamental role in the functioning of the neostriatum by modulating the spiking of striatal projection neurons with great efficacy. The powerful and strongly divergent output of the GABAergic interneurons neurons suggests that modulation of their activity may be particularly effective at controlling the functioning of the entire neostriatal circuitry. Acetylcholine is one of the main modulators of striatal functioning. The effects of acetylcholine on fast-spiking (FS) GABAergic interneurons were studied with whole-cell recording in an in vitro slice preparation. Acetylcholine exerted two distinct effects on fast-spiking interneurons. Acetylcholine directly depolarized FS interneurons by acting on nondesensitizing soma-dendritic nicotinic receptors. In addition, acetyl-
\end{abstract}

choline attenuated the GABAergic inhibition of projection neurons by fast-spiking interneurons through activation of presynaptic muscarinic receptors. It is suggested that the nicotinic excitation of FS interneurons may play an important role in translating the effect of the brief behaviorally contingent cessation of firing of the tonically active cholinergic interneurons to the output neurons of the neostriatum. In contrast, the muscarinic presynaptic inhibitory mechanism may be engaged primarily during longer-lasting elevations of extracellular acetylcholine levels.

Key words: fast-spiking interneuron; neostriatum; GABA; acetylcholine; nicotinic receptor; muscarinic receptor; presynaptic inhibition; cholinergic interneuron; tonically active neurons
Cholinergic signaling in the neostriatum is an important modulator of the activity of the basal ganglia and has long been recognized to act in a complex, and in many ways antagonistic, manner with respect to dopaminergic transmission in striatal function. Most of the studies concerning the effects of acetylcholine $(\mathrm{ACh})$ on the intrinsic properties of neostriatal neurons have concentrated on the medium spiny (MS) projection neurons. Complex modulation of the overall excitability, as well as the voltage dependence, kinetic properties, and/or maximum conductance of various potassium, calcium, and sodium channels of striatal spiny neurons has been demonstrated and has led to insightful predictions about the functional consequences of these neuromodulatory actions (Akins et al., 1990; Plenz and Kitai, 1998). Furthermore, complex effects of ACh on synaptic transmission and plasticity in the neostriatum have been described previously (Sugita et al., 1991; Calabresi et al., 1998, 1999). In general, however, it has been difficult to provide a mechanistic explanation for the systems level effects of cholinergic manipulations in the neostriatum based on this information alone (Calabresi et al., 2000). Part of the difficulty in bridging these cellular and network level phenomena may arise from the additional complexities introduced by cholinergic effects on striatal neuronal populations in addition to the MS neurons.

Received July 30, 2001; revised Oct. 23, 2001; accepted Oct. 25, 2001.

This research was supported in part by National Institutes of Health Grants $\mathrm{MH}$ 58885 and NS 34865 and a Busch Biomedical Support Grant from Rutgers University. We thank E. Borók and F. Shah for excellent technical assistance and Dr. J. C. Magee for providing a slice preparation protocol for adult animals.

Correspondence should be addressed to Dr. James M. Tepper, Center for Molecular and Behavioral Neuroscience, Program in Cellular and Molecular Biodynamics, Rutgers University, 197 University Avenue, Newark, NJ 07102. E-mail: tepper@axon.rutgers.edu.

T. Koós present address: Section of Neurobiology, Yale University School of Medicine, 333 Cedar Street, P.O. Box 208001, New Haven, CT 06520-8001.

Copyright (C) 2002 Society for Neuroscience $0270-6474 / 02 / 220529-07 \$ 15.00 / 0$
Recent anatomical and electrophysiological studies suggest that, despite their relatively small number, aspiny GABAergic interneurons have a powerful effect on the overall functioning of the neostriatum (Bolam et al., 1983; Kita and Kitai, 1988; Kita et al., 1990; Jaeger et al., 1994; Kawaguchi et al., 1995; Gerfen and Wilson, 1996; Kita, 1996). At least some of these interneurons are known to receive cholinergic synaptic input (Chang and Kita, 1992). Consequently, the control of these neurons and/or their synaptic interactions with projection neurons by ACh may be essential in determining the overall effect of $\mathrm{ACh}$ on the output of the neostriatum. In the current study, the effects of ACh on fast-spiking (FS) interneurons and their synaptic interaction with MS neurons were investigated using single and paired whole-cell recordings in slices of the rat neostriatum.

\section{MATERIALS AND METHODS}

Electrophysiology. Slices were from obtained from either 13- to 30-d-old juvenile or adult (110-208 gm) Sprague Dawley rats. All procedures were performed with the approval of the Rutgers University Institutional Animal Care and Use Committee and in accordance with the NIH Guide to the Care and Use of Laboratory Animals. Slices were prepared and maintained with the following modifications of previously described methods (Koós and Tepper, 1999). The Ringer's solution used for recording and maintaining the slices contained (in $\mathrm{mM}$ ): $125.0 \mathrm{NaCl}, 2.5$ $\mathrm{KCl}, 2.0 \mathrm{CaCl}_{2}, 1.0 \mathrm{MgCl}_{2}, 25.0 \mathrm{NaHCO}_{3}, 1.25 \mathrm{NaH}_{2} \mathrm{PO}_{4}, 25.0$ glucose, $\mathrm{pH}$ 7.3-7.4, 1.0 ascorbate, 3.0 pyruvate, and 0.4 myo-inositol. In some cases, ascorbate, Pyruvate, and myo-inositol were omitted and the concentrations of $\mathrm{CaCl}_{2}, \mathrm{MgCl}_{2}$, and glucose were changed to $2.5,1.5$, and $9.0 \mathrm{~mm}$ respectively. Adult slices were prepared in ice-cold (approximately $-4^{\circ} \mathrm{C}$ ) modified solution containing (in $\mathrm{mm}$ ): 225.0 sucrose, $2.5 \mathrm{KCl}$, $0.5 \mathrm{CaCl}_{2}, 7.0 \mathrm{MgCl}_{2}, 28.0 \mathrm{NaHCO}_{3}, 1.25 \mathrm{NaH}_{2} \mathrm{PO}_{4}, 7.0$ glucose, 1.0 ascorbate, and 3.0 pyruvate. The animals were transcardially perfused with $\sim 60 \mathrm{ml}$ of the modified Ringer's solution at a rate of $\sim 20 \mathrm{ml} / \mathrm{min}$ before slice preparation.

Whole-cell current-clamp recordings were performed as described previously (Koós and Tepper, 1999). For voltage-clamp recordings, the internal solution contained (in $\mathrm{mM}$ ): $140.0 \mathrm{CsCl}, 2.0 \mathrm{MgCl}_{2}, 10.0$ 
HEPES, 3.0 Na 2 ATP, $0.3 \mathrm{Na}_{3} \mathrm{GTP}, 10.0$ EGTA, and usually 5 QX-314 (Sigma, St. Louis, MO), pH 7.2-7.3. Biocytin (0.1-0.5\%) was also added to the solution in most cases. Recordings were made with a Neurodata Instruments (New York, NY) IR-283 current-clamp and an EPC-9 (Heka Elektronik, Lambrecht/Pfalz, Germany) voltage-clamp amplifier. Voltage-clamp recordings were obtained with 3.5-5 $\mathrm{M} \Omega$ pipettes. The membrane potential of the MS neurons was clamped at $-70 \mathrm{mV}$. Series resistance compensation of $30-60 \%$ was used, and recordings were filtered online with a second-order Bessel filter at $1 \mathrm{kHz}$ and digitized at $10 \mathrm{kHz}$. Voltage-clamp experiments were performed at room temperature. During paired recording experiments, the presynaptic interneurons were stimulated at $0.5 \mathrm{~Hz}$. At the completion of the experiments, slices were processed as described previously (Koós and Tepper, 1999) to visualize biocytin-stained neurons (Horikawa and Armstrong, 1988).

Pharmacology. ACh chloride, AP-5, atropine sulfate, bicuculline methochloride, carbamylcholine chloride (carbachol), disodium-CNQX, muscarine chloride, mecamylamine hydrochloride (MEC), methyllycaconitine citrate (MLA), nicotine di- $d$-tartarate, and pirenzepine dihydrochloride were obtained from Research Biochemicals (Natick, MA). CGP 55845A was a gift from Ciba-Geigy (Basel, Switzerland). Drugs were freshly dissolved in Ringer's solution as indicated, except for CGP $55845 \mathrm{~A}, \mathrm{MLA}$, and muscarine, which were stored at $-20^{\circ} \mathrm{C}$ in aqueous stock solutions. Drugs were applied in the perfusion medium or locally, via a pipette $(<2 \mu \mathrm{m}$ tip) using a Picospritzer (General Valve, Fairfield, NJ). Drugs were ejected at $10-30 \mathrm{sec}$ intervals with 10 psi and $40-100$ msec duration pressure pulses. The pipettes were positioned 30-70 $\mu \mathrm{m}$ $(51.7 \pm 5.4 \mu \mathrm{m} ; n=6)$ from the soma of the recorded neurons and at least $10 \mu \mathrm{m}$ beneath the surface of the slice. Drug concentrations for bath application were selected with reference to receptor affinities (Doods et al., 1987). Drug concentrations for pressure applications were determined empirically to provide specific and sufficiently strong effects, consistent with previous reports (Guo et al., 1998).

As a positive control for the effectiveness of MLA, a pressure application experiment was performed using $400 \mu \mathrm{m}$ coronal slices of the dorsal hippocampus (see Results). The experiment was conducted subsequent to the collection of all striatal data using the same batch of stock solution of MLA.

Data analysis. For IPSC amplitude measurements, window averages of the recorded current were calculated at two positions individually for each trace. The window averages were calculated as the arithmetic mean of the digitized current values within a time window of $4.6 \mathrm{msec}$ duration. The IPSC amplitude was defined as the difference between two window averages, with one window placed just after the peak of the IPSC and another before the onset of the response. The two time windows were separated exactly by $16.6 \mathrm{msec}$ relative to each other to eliminate line noise. Background noise distribution was determined the same way as IPSC amplitudes but with the two averaging windows shifted to $\sim 30$ msec before the elicited response (Stricker and Redman, 1994; Stricker et al., 1996). The coefficient of variation (CV) of IPSCs was calculated by dividing the SD of the IPSC amplitude by the mean amplitude. Population data are reported as mean $\pm \mathrm{SE}$.

\section{RESULTS}

\section{Nicotinic depolarization of FS interneurons}

FS interneurons were targeted on the basis of their appearance with differential interference contrast microscopy under infra-red transillumination and were identified electrophysiologically (Kawaguchi, 1993; Kawaguchi et al., 1995; Koós and Tepper, 1999). All FS interneurons were strongly hyperpolarized (less than $-70 \mathrm{mV}$ ) and silent at rest. FS interneurons fired narrow action potentials and exhibited high maximal firing frequencies $(\sim 200 \mathrm{~Hz})$ in response to current injection, as shown in Figure 1. In addition, constant depolarizing current injections induced an irregular bursty firing pattern (Fig. $1 B$ ). The morphological properties of biocytin-stained FS interneurons corresponded to previous descriptions of this cell type ( $n=5$; data not shown) (Kita et al., 1990; Kawaguchi, 1993; Kita, 1993; Kubota et al., 1993; Kawaguchi et al., 1995; Koós and Tepper, 1999).

Ten of 11 FS interneurons responded to local $(n=8)$ or bath $(n=3)$ application of cholinergic agonists. For local pressure application experiments, nicotinic agonists $(n=8)$ were applied,
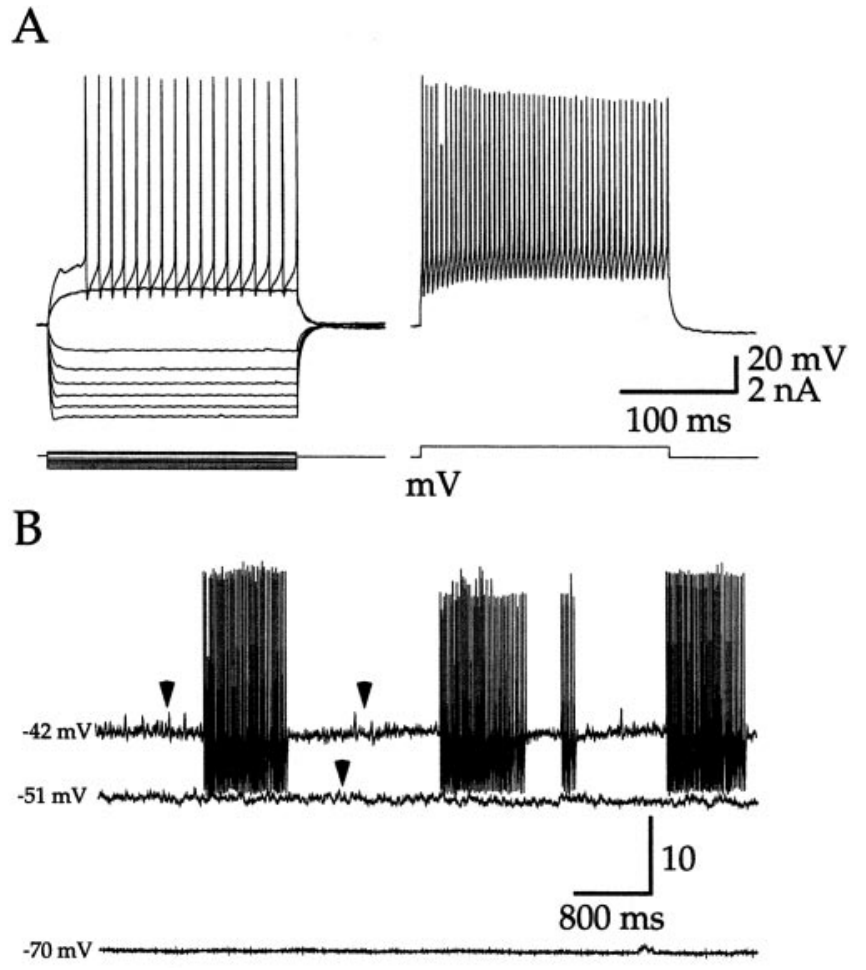

Figure 1. Characteristic electrophysiological properties of striatal FS interneurons recorded in whole-cell mode in vitro. $A$, Responses of a typical FS interneuron to intracellularly injected current pulses. Note the high maximal firing rate $(\sim 200 \mathrm{~Hz}$; right panel $)$. B. Typical response of an FS interneuron to constant depolarizing current injection of varying amplitudes. All FS interneurons were hyperpolarized and silent at rest. Depolarizing current injection elicited subthreshold membrane potential fluctuations (arrowheads), which were always absent at rest and could also induce episodes of firing. Firing epochs occurred at irregular intervals but exhibited stable intraburst frequencies. The firing pattern was stable over the duration of the depolarization $(>10 \mathrm{sec})$. Action potentials truncated as a result of aliasing.

including $20 \mathrm{~mm}$ carbachol $(n=4), 3-5 \mathrm{~mm} \mathrm{ACh}(n=3)$, or 250 $\mu \mathrm{M}$ nicotine $(n=1)$.

Locally applied cholinergic agonists depolarized FS interneurons by $7.1 \pm 1.8 \mathrm{mV}(n=8$; range of 3.4-13.7 mV) (Fig. $2 A)$. The response to pressure-applied cholinergic agonists could be completely blocked by bath application of the selective nicotinic receptor antagonist MEC at concentrations of $1-10 \mu \mathrm{M}(n=4)$, as shown in Figure $2 A$. In one other FS interneuron, $1 \mu \mathrm{M}$ MEC reduced the depolarization by $92 \%$. Partial reversal of the MEC blockade was obtained by washing for $40 \mathrm{~min}(5 \mu \mathrm{M} ; n=1)$ (Fig. $2 A)$. To further characterize the receptor subtype underlying the nicotinic depolarization, MLA, a selective antagonist of the $\alpha 7$ subunit containing type 1 nicotinic receptors, was applied at 100 $\mathrm{nM}$, a concentration sufficient to completely block these receptors, as well as certain $\beta 2$ subunit-containing (type 2) receptors (Alkondon and Albuquerque, 1993). MLA (100 nM; $n=3$ ) had little or no effect on the depolarization evoked by pressure application of nicotinic agonists $(n=3)$, including ACh (3-5 mM; $n=$ 2) or carbachol ( $20 \mathrm{mM} ; n=1)$, as illustrated for one neuron in Figure $2 B$. These responses were all blocked by the subsequent application of 1-10 $\mu \mathrm{M}$ MEC (Fig. $2 B$ ). To demonstrate the efficacy of MLA, the effects of the drug were tested on carbacholinduced depolarization of hippocampal stratum radiatum interneurons, a cell type known to express type 1 nicotinic receptors 
A

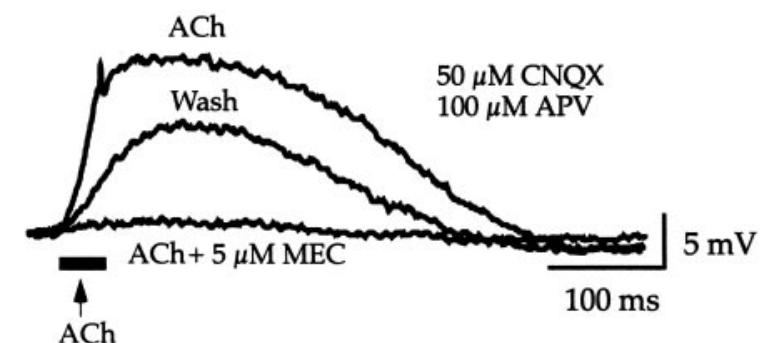

$\mathrm{C}$

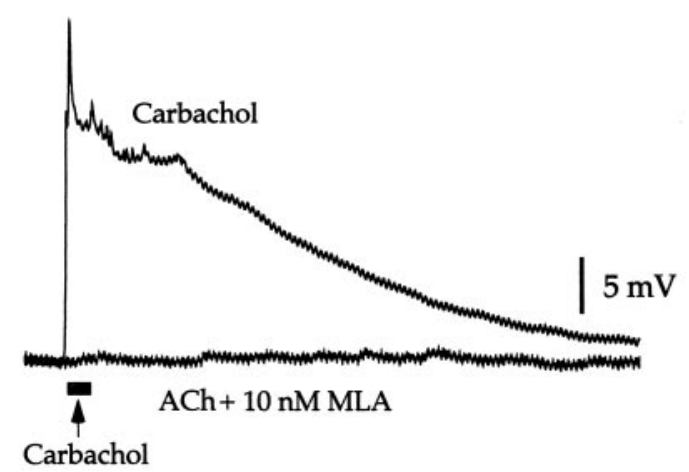

B

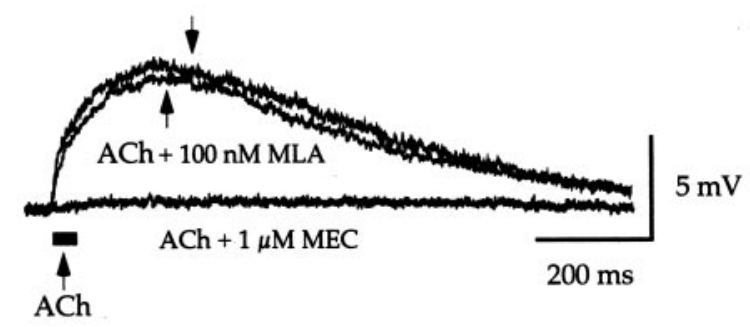

$\mathrm{D}$

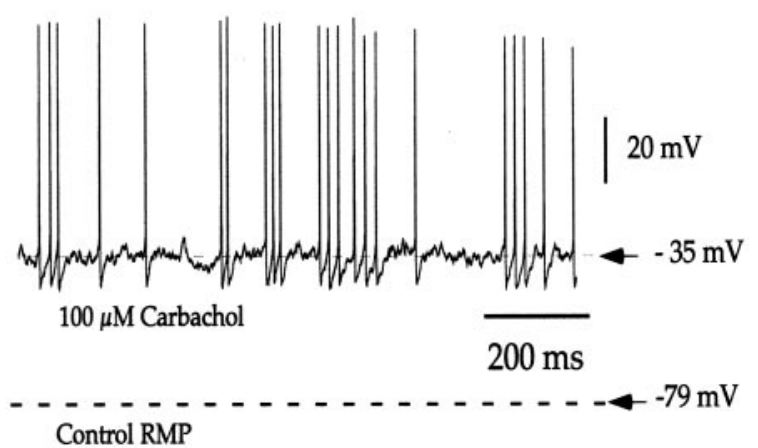

Figure 2. Nicotinic receptor-mediated responses of FS interneurons. A, Local pressure application of ACh (5 mM; 40 msec duration; thick bar) induced a depolarization (ACh) that was blocked by bath application of $5 \mu \mathrm{M}$ MEC. There was partial recovery after washing for 40 min. Note that the experiment was conducted in the presence of CNQX and APV. B, Local pressure application of $5 \mathrm{~mm}$ ACh (thick bar) evoked a depolarization (ACh) that was primarily unaffected by bath application of $100 \mathrm{nM}$ MLA but was almost completely blocked by subsequent application of $1 \mu \mathrm{M}$ MEC. $C$, As a positive control, the effectiveness of MLA was tested on the nicotinic depolarization evoked by local pressure application of $5 \mathrm{~mm}$ carbachol (thick bar) on a hippocampal stratum radiatum interneuron. The strong depolarization and firing of action potentials (top trace; action potentials truncated as a result of averaging) were completely blocked by bath application of $10 \mathrm{nM}$ MLA, demonstrating the potency of the antagonist. $D$, Bath application of $100 \mu \mathrm{M}$ carbachol depolarized a silent FS interneuron by $\sim 44 \mathrm{mV}$ and induced irregular bursty firing. The firing was maintained for the duration of the drug application (>10 $\mathrm{min})$.

(Frazier et al., 1998). On these neurons, MLA was shown to be fully potent at the expected effective concentration (Frazier et al., 1998), as shown in Figure $2 C$.

The effect of bath application of cholinergic agonists was also tested on four FS interneurons, all of which were depolarized by agonist administration. Application of carbachol $(40 \mu \mathrm{M} ; n=1)$ or nicotine (100 $\mu \mathrm{M}, n=2 ; 200 \mu \mathrm{M}, n=1)$ depolarized the interneurons by $15(10.3 \pm 2.6)$ or $9.5 \mathrm{mV}$, respectively. The depolarization was stable for the duration of the drug application $(>10 \mathrm{~min})$ and could be fully reversed during washing with normal Ringer's solution $(n=1)$ or be blocked by $1-5 \mu \mathrm{M}$ MEC $(n=3)$. In addition, a higher concentration of carbachol $(100 \mu \mathrm{M})$ could induce an MEC-sensitive, irregular, bursty firing in FS interneurons $(n=2)$ (Fig. $2 D)$. The pattern of firing induced by carbachol was similar to the one evoked by constant current injection (Figs. $1 B, 2 D$ ). In contrast, muscarine did not have a measurable effect on the resting membrane potential of FS interneurons and, although this was not examined systematically, did not appear to alter the latency, threshold, duration, or afterhyperpolarization of action potentials evoked by intracellular current injection in these neurons (Fig. 3, 4A, bottom traces).

To control for the possibility that the nicotinic responses were mediated indirectly through the induced release of glutamate (McGhee et al., 1995; Radcliffe et al., 1999), in four cases, the response to bath $(n=2)$ or local pressure application $(n=2)$ of cholinergic agonists was examined in the presence of the ionotropic glutamate receptor antagonists CNQX $(50 \mu \mathrm{M})$ and APV
$(100 \mu \mathrm{M})$. The presence of these drugs did not block or otherwise affect the nicotinic depolarization of FS interneurons (Fig. 2A).

\section{Presynaptic muscarinic inhibition of synaptic transmission between FS interneurons and MS neurons}

The effects of muscarinic agonists on synaptic transmission between FS interneurons and MS neurons were tested on 10 synaptically connected cell pairs. Two of the 10 pairs involved the same FS interneuron paired with a different spiny neuron. Four pairs were recorded in slices obtained from young animals (average of $21 \pm 2.9 \mathrm{~d}$; range of 14-28 d) and six pairs in adults (average of 151.3 $\pm 17.1 \mathrm{gm}$; range of 110-208 gm). There was no statistically significant difference between the age groups with respect to the mean IPSC amplitudes or CV of the IPSCs (unpaired $t$ test; $p>0.8$ and $p>0.5$, respectively), and therefore the data were pooled.

Single action potentials of FS interneurons elicited IPSCs in synaptically connected MS neurons with a generally high but variable fidelity (Fig. 3). In most pairs, the failure rate was $<1 \%$ ( $n=4$ pairs; $\sim 200$ trials). One pair exhibited a slightly higher failure rate of $4.9 \%$, and, in one pair, a failure rate of $33.3 \%$ was observed. The mean peak IPSC amplitude was $-212.6 \pm 73 \mathrm{pA}$ under control conditions (range of $-21.9-499.3 \mathrm{pA} ; n=9$ pairs; 20-200 traces averaged for each pair at $-70 \mathrm{mV}$ ). A control amplitude could not be measured for one of the 10 pairs in which the recording was initiated in the presence of muscarine. The 


\section{Postsynaptic MS neuron}

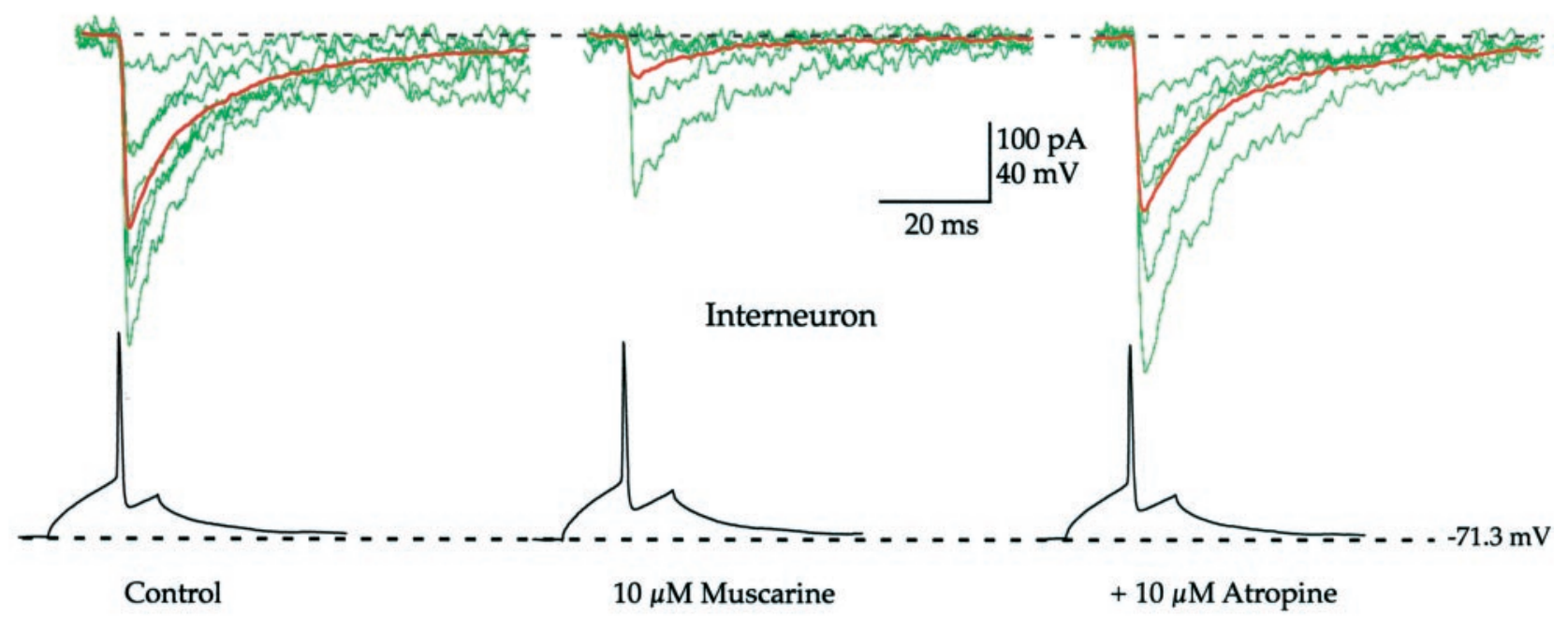

Presynaptic FS

Figure 3. Muscarinic receptor-mediated inhibition of synaptic transmission between FS interneurons and MS neurons. Representative example of recording from a synaptically connected pair of an FS interneuron and an MS neuron. Single action potentials of the FS interneuron elicited IPSCs ( green sweeps) with variable amplitudes (left). Bath application of $10 \mu \mathrm{M}$ muscarine strongly reduced the average amplitude of the postsynaptic response without affecting passive or active properties of the FS interneuron. Coapplication of $10 \mu \mathrm{M}$ atropine reversed the effect of muscarine (right). Red traces are population means. IPSCs are inward because of chloride loading (see Materials and Methods).

trial-to-trial variance of the IPSC amplitude for individual pairs far exceeded the stochastic background fluctuation of the recording resulting from spontaneous synaptic activity and other sources, suggesting the involvement of multiple release sites (see below) (Figs. 3, 4A). The decaying phase of the IPSC could be fitted with a single exponential with a time constant of $12.1 \pm 0.7$ $\operatorname{msec}\left(n=4\right.$ pairs; $\left.20-25^{\circ} \mathrm{C}\right)$.

Bath application of muscarine $(2.5-10 \mu \mathrm{M})$ reduced the mean IPSC amplitude elicited by single action potentials of FS interneurons to $13.7-54.3 \%$ of their original amplitude $(n=8$ pairs; 20-200 IPSCs for each pair) (Fig. 3). In another pair, $10 \mu \mathrm{M}$ carbachol reduced the average IPSC amplitude to $38.5 \%$ of control. The reduction was statistically significant for each pair (Mann-Whitney $U$ test; $p<0.0001$ ). Because each neuron was only tested at a single drug concentration, a dose-response relationship of the inhibition could not be determined for individual pairs. However, linear regression of the percentage of control IPSC amplitude in muscarine against the concentration of the drug across the eight pairs resulted in a statistically significant linear fit with $R^{2}=-0.669(F=10.13 ; p=0.024)$, demonstrating a dose dependence of the muscarinic effect.

Application of the nonselective muscarinic antagonist atropine (5-10 $\mu \mathrm{M})$ blocked $79.1 \pm 10.9 \%$ of the muscarine-induced IPSC amplitude reduction ( $n=5$ pairs; 50-200 IPSCs for each pair) (Fig. 3). The blockade was statistically significant (Mann-Whitney $U$ test; $p<0.0001$ for all five pairs) and was complete in all but two cases in which sufficiently long drug duration application was not possible (Fig. 3). To further characterize the muscarinic receptor subtype, the effect of pirenzepine, a selective $M_{1} / M_{4}$ receptor antagonist (Doods et al., 1987), was also tested. Pirenzepine at 100-200 nM significantly increased the IPSC amplitude measured in the presence of muscarine $(2.5-10 \mu \mathrm{M} ; 59 \pm 16.8 \%$; $n=3$ pairs; Mann-Whitney $U$ test; $p<0.005$ for each pair). The pirenzepine-induced amplitude increase represented the recov- ery of approximately half $(47.6 \pm 15.7 \%)$ of the muscarinic inhibition $(n=2)$.

No formal quantal analysis was attempted on the data because of the relatively small number of IPSCs recorded under each condition (Stricker and Redman, 1994; Stricker et al., 1996). However, in most cases, muscarinic receptor stimulation appeared to shift the distribution of the IPSC amplitudes by increasing the relative frequency of smaller amplitude responses without altering the apparently discreet amplitude levels themselves (Fig. $4 A$ ), suggestive of a presynaptic mechanism of inhibition. The possibility of a presynaptic locus of action for muscarine was further examined by analyzing the drug-induced change in the probability of failures and the $\mathrm{CV}$. Failure rates were calculated only for those pairs for which the minimal response amplitude was sufficiently large to reliably discriminate them from the background noise. The average control failure rate for these pairs was $0.46 \pm 0.4 \%$ ( $n=4$ pairs $)$. Application of muscarine significantly increased the failure rate to $12.7 \pm 4.4 \%\left(n=4 ; \chi^{2}\right.$ test; $p<0.025$ for each pair). In addition to the change in failure rate, the decrease in the mean IPSC amplitude during muscarine application was paralleled by an increase of the CV of the IPSCs (Fig. $4 B)$. The mean $\mathrm{CV}$ for the nine pairs under control conditions was $0.53 \pm 0.08$. After reaching maximal effect, muscarine significantly increased the CV to $0.97 \pm 0.12$, or $183 \%$ of control $(n=$ 9 pairs; 20-200 IPSCs for each pair; $F$ test; $p<0.0001$ for each pair). Four of the seven pairs tested with a nonparametric but less sensitive test for the equality of variances (Wald-Wolfowitz runs test) also showed a significant difference at $p<0.005$. Linear regression analysis revealed a strong correlation, with $R^{2}=0.91$ $(F=53.1 ; p=0.0008)$ between the magnitude of reduction of the average IPSC amplitude and the relative change of the CV across the pairs tested (Fig. $4 C$ ). The validity of the $\mathrm{CV}$ method depends on the relative magnitudes of the noise- and signal-related variance of the PSC amplitudes. In the recorded pairs, $0.19-4.44 \%$ of 
A
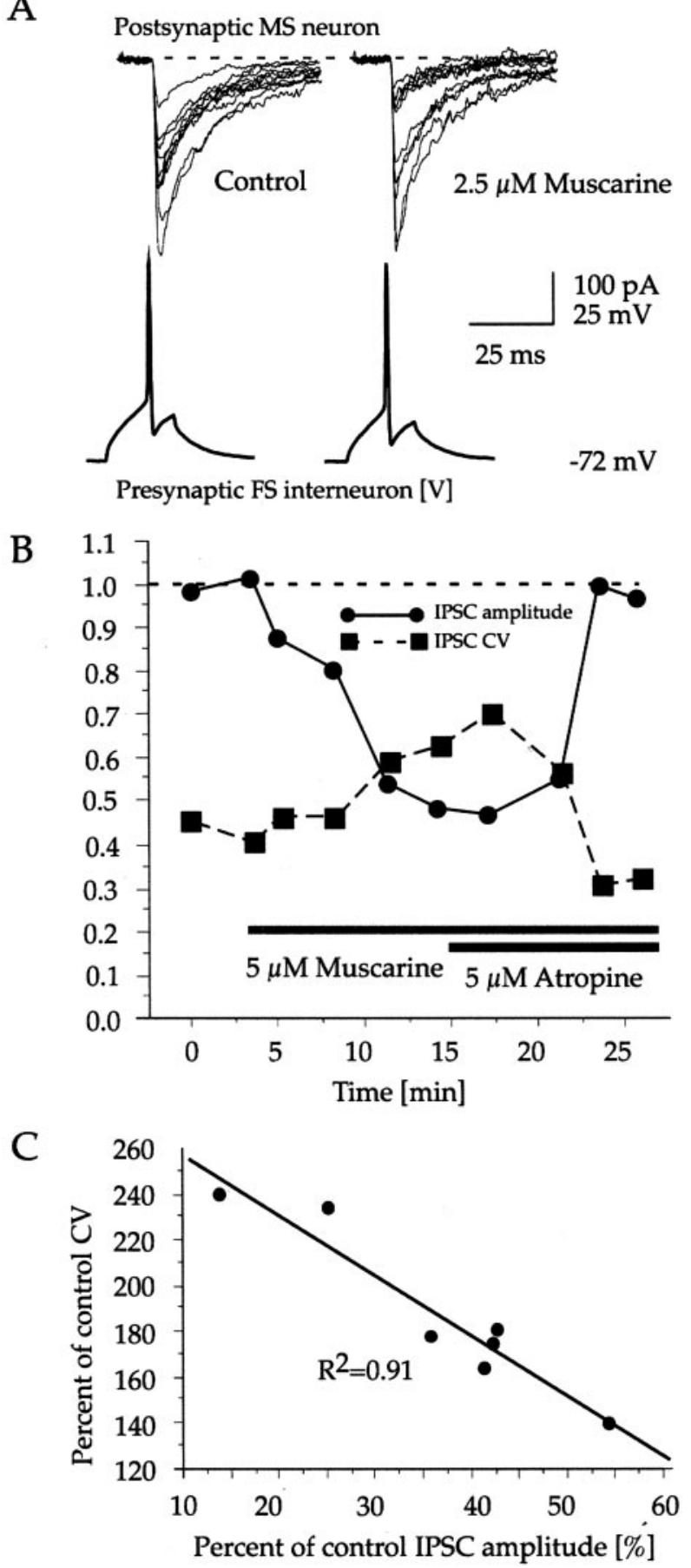

Figure 4. Muscarinic modulation of GABAergic IPSCs between FS and MS neurons is presynaptic. $A$, Muscarine induced change in the amplitude distribution of IPSCs. Ten consecutive traces are shown for control condition (left) and after the application of $2.5 \mu \mathrm{M}$ muscarine (right). Approximately the same amplitude levels were observed under the two conditions, but there is clear shift in relative frequencies away from the larger and toward the smaller and intermediate response amplitudes induced by muscarine. To prevent the complete disappearance of larger amplitude responses, the traces shown were recorded before the full inhibitory effect of muscarine developed ( $\sim 30 \%$ inhibition). $B$, Correspondence between the reduction in IPSC amplitude and increase of CV for a pair of FS and MS neurons. Each point represents the mean amplitude and $\mathrm{CV}$ for $\sim 20$ consecutive responses. $C$, Linear regression of the percentage of change of $\mathrm{CV}$ induced by muscarine against the percentage of reduction of the average IPSC amplitude for seven pairs. the variance of the IPSC amplitudes was attributable to background noise, as determined by measuring the current amplitude fluctuation just before the evoked IPSCs (see Materials and Methods).

An indirect mediation of the muscarinic reduction of the IPSCs by another neurotransmitter system could not be completely excluded in this study. However, the involvement of $\mathrm{GABA}_{\mathrm{B}}$ receptors was ruled out because the high-affinity $\mathrm{GABA}_{\mathrm{B}}$ receptor antagonist CGP 55845A ( 1 or $2 \mu \mathrm{M} ; n=2$ ) failed to prevent the inhibitory effects of muscarine on the IPSCs (data not shown). Although not studied systematically, CGP 55845A did not appear to affect the IPSC amplitude or alter simple pairedpulse ratios at 300 or $500 \mathrm{msec}$ interstimulus intervals.

In three pairs in which muscarinic presynaptic inhibition was demonstrated, the effect of bath application of nicotine $(100 \mu \mathrm{M}$, $n=2 ; 200 \mu \mathrm{M}, n=1)$ was also tested. All three FS interneurons were depolarized by nicotine $(9.66 \pm 2.33 \mathrm{mV})$, demonstrating that functional nicotinic soma-dendritic receptors and muscarinic presynaptic receptors coexist in at least some neostriatal FS interneurons.

\section{DISCUSSION}

The present study is the first to examine the effects of ACh on neostriatal GABAergic FS interneurons. The main findings are that ACh affects FS interneurons both postsynaptically, resulting in a powerful nicotinic excitation, and presynaptically, resulting in a muscarinic presynaptic inhibition of GABAergic synaptic transmission between FS interneurons and spiny projection neuron. Considering the powerful effect of these interneurons on the activity of the projection neurons (Koós and Tepper, 1999), the cholinergic control of these interneurons is expected to have a significant effect on the overall activity of the neostriatum.

ACh led to substantial excitation of FS interneurons via nicotinic receptors. The nicotinic excitation exhibited no apparent desensitization and produced depolarization sufficient to elicit spiking in previously silent interneurons. The nicotinic excitation appears to be a direct postsynaptic effect, independent of glutamatergic afferents (McGhee et al., 1995; Radcliffe et al., 1999), because it persisted in the presence of blockade of NMDA and non-NMDA receptors. The blockade of the nicotinic depolarization of FS interneurons by MEC, the insensitivity of the response to high concentrations of MLA, and the sustained response to bath application of nicotinic agonists strongly suggest that the response is not mediated by the exclusively $\alpha 7$ subunit-containing type 1 receptors (Alkondon and Albuquerque, 1993; Zoli et al., 1998). Some type 2 receptors containing $\beta 2$ subunits can also be ruled out (Zoli et al., 1998); however, it is possible that, depending the subtypes of heteromeric $\alpha$ subunits involved, a $\beta 2$ containing receptor might still be involved.

One of the eleven tested FS interneurons did not respond to either bath $(20 \mu \mathrm{M})$ or pressure $(20 \mathrm{~mm})$ application of carbachol. The intrinsic properties of this neuron did not appear to be different from those of Ach-responsive FS interneurons. This finding may reflect a heterogeneity among FS neurons, possibly corresponding to the previously noted morphological diversity of this neuron type (Kita et al., 1990; Kawaguchi, 1993; Kubota and Kawaguchi, 1993; Kawaguchi et al., 1995; Koós and Tepper, 1999).

Monosynaptic innervation of parvalbumin-positive interneurons by choline acetyltransferase-positive terminals has been demonstrated in the neostriatum (Chang and Kita, 1992). Be- 
cause parvalbumin-positive neurons most likely correspond to FS interneurons (Kawaguchi, 1993; Kita, 1993; Kawaguchi et al., 1995; Koós and Tepper, 1999), FS interneurons receive monosynaptic input from cholinergic interneurons. Surprisingly, however, in a few preliminary experiments, we were unable to reliably elicit nicotinic EPSPs in FS interneurons using extracellular field stimulation. The reasons for this failure are unclear, but several possibilities exist, including that our stimulation failed to recruit a sufficient number of cholinergic afferents, that the cholinergic terminals were compromised in some way, or possibly that simultaneous release of other neurotransmitters effectively attenuated $\mathrm{ACh}$ release. It is possible, however, that, in contrast to the slice preparation, in the in vivo situation volume transmission of ACh may be sufficient to activate the receptors extrasynaptically.

The second major effect of ACh on FS interneurons was inhibition of the FS-evoked IPSC in MS neurons. Cholinergic inhibition of GABAergic transmission has been demonstrated previously in the neostriatum (Sugita et al., 1991; Szabo et al., 1998). Those studies showed that GABAergic IPSPs evoked by extracellular stimulation were reduced in amplitude by the application of muscarinic agonists. The present study confirms and extends these previous findings. In particular, the use of the dual recording technique allowed the identification of the afferent source of at least one ACh-modulated GABAergic input of MS neurons as the FS interneurons.

The presynaptic locus of action of ACh was inferred from a significant change of the $\mathrm{CV}$ of the IPSC. Calculation of the $\mathrm{CV}$ changes the variance introduced by noise and therefore can result in an artifactual appearance of a change in the overall $\mathrm{CV}$ of the IPSC, even in the case of a purely postsynaptic reduction of the evoked response (Clements and Silver, 2000). To control for this possibility, the relative contribution of noise to the total variance was estimated. The noise variance was a only a small fraction $(0.19-4.44 \%)$ of the total signal variance. Therefore, the near doubling of the $\mathrm{CV}$ under muscarinic stimulation cannot be a result of the scaling of the noise-related variance and hence reflects a change in the IPSC amplitude distribution and demonstrates a presynaptic mechanism of action for $\mathrm{ACh}$, consistent with the increased IPSP failure rate.

Cholinergic regulation of neostriatal GABA release has also been studied neurochemically. Nonselective cholinergic agonists have been shown to increase basal GABA overflow in vitro through nicotinic receptors (Limberger et al., 1986). In contrast, GABA overflow induced by electrical stimulation (Sugita et al., 1991) or high extracellular $\mathrm{K}^{+}$(Marchi et al., 1990) was found to be inhibited by ACh through muscarinic receptors. These opposite effects of cholinergic stimulation on GABA release in the stimulated and unstimulated conditions may be explained by the dual regulation of the FS interneuron through nicotinic and muscarinic mechanisms reported in the present paper. Considering that FS interneurons are hyperpolarized and inactive in vitro (Fig. 1B, bottom trace) (Kawaguchi, 1993; Koós and Tepper, 1999) and assuming that the rate of action potential-independent spontaneous release of GABA from their terminals is low, it would be expected that, in the presence of a nonselective agonist, the GABA release-inducing effect of nicotinic stimulation would predominate over presynaptic muscarinic inhibition. Conversely, when FS interneurons are activated to release GABA, the stimuli may excite the FS interneurons to such levels such that additional nicotinic stimulation produces only a relatively minor further increase in firing rate and consequent GABA release, and therefore the inhibitory presynaptic effect of the muscarinic receptors dominates. The results of the present study, however, cannot in themselves account for the finding that nicotinic receptormediated increase of GABA release persists in TTX (Limberger et al., 1986; Radcliffe et al., 1999). Therefore, nicotinic receptors may also be present on some GABAergic presynaptic terminals. Although these presynaptic receptors may be expressed on FS interneurons, it is also possible that the TTX-insensitive nicotinic receptor-mediated GABA release originates from other striatal GABAergic terminals.

The dual cholinergic control of the inhibition of MS neurons by FS interneurons described here suggests a complex regulation of these interneurons by ACh. The activation of soma-dendritic nicotinic receptors should increase the feedforward inhibition of spiny projection neurons, whereas presynaptic muscarinic receptors are expected to exert the opposite effect. The balance between these opposing effects will depend on the magnitude and timing of changes in ACh release. Neostriatal ACh release is under a complex regulation involving dopaminergic, glutamatergic, GABAergic, and other inputs inducing both rapid and slow changes in ACh levels (Aosaki et al., 1994, 1995; Smolders et al., 1995; DeBoer and Abercrombie, 1996; DeBoer et al., 1996; Abercrombie and DeBoer, 1997). These patterns of ACh release could be differentially registered by muscarinic and nicotinic receptors of FS interneurons. In particular, there is evidence that resting ACh levels do not tonically inhibit GABA release through muscarinic receptors in the neostriatum (Smolders et al., 1995). Because ACh release increases significantly during cortical activation (Timmerman and Abercrombie, 1996; Abercrombie and DeBoer, 1997), it is possible that presynaptic muscarinic receptors on FS interneuron terminals are activated specifically during periods of increased behavioral arousal.

On a much faster time scale, dramatic behaviorally contingent regulation of $\mathrm{ACh}$ release has been demonstrated by recording the firing of physiologically identified cholinergic interneurons in the primate neostriatum (Apicella et al., 1991; Aosaki et al., 1994, 1995; Schultz et al., 1995). It has been shown that putative cholinergic interneurons (tonically active neurons, or TANs) cease firing for a short $(<300 \mathrm{msec})$ period when the animal is presented with a behaviorally relevant sensory cue (Aosaki et al., 1994, 1995). It is unclear, however, how this brief change in ACh release is registered by the neostriatal spiny neurons. Because of the short duration of the pause in the firing of TANs, a temporally correlated effect would require the transmission of this signal by a sufficiently fast postsynaptic signaling mechanism. Although direct membrane-delimited signaling pathways of muscarinic receptors, including those regulating MS neurons (Howe and Surmeier, 1995), may be sufficiently rapid to reverse partially during these brief reductions of ACh release (Zhou et al., 1997), the nondesensitizing nicotinic receptors of FS interneurons are likely to follow these events more effectively and with more temporal specificity. Because FS-spiny synaptic transmission is primarily $\mathrm{GABA}_{\mathrm{A}}$ mediated (Koós and Tepper, 1999) and therefore also fast, it is possible that disfacilitation of FS interneurons plays a crucial role in mediating the pause signal of cholinergic interneurons to the rest of the striatal circuitry, in particular to the spiny neuron, with great temporal fidelity. The reduced activity of these interneurons would in turn cause disinhibition of MS neurons, inducing increased output from the neostriatum. Consistent with this hypothesis, an increase in firing rate of some MS neurons is observed during the same phase of the behavioral task during which the pause of firing of TANs occurs (Hikosaka et al., 1989). Disinhibition of MS neurons in response to the detection of 
relevant stimuli may serve an important function in the generation of the behavioral response.

\section{REFERENCES}

Abercrombie ED, DeBoer P (1997) Substantia nigra D1 receptors and stimulation of striatal cholinergic interneurons by dopamine: a proposed circuit mechanism. J Neurosci 17:8498-8505.

Akins PT, Surmeier DJ, Kitai ST (1990) Muscarinic modulation of a transient $\mathrm{K}^{+}$conductance in rat neostriatal neurons. Nature $344: 240-242$.

Alkondon M, Albuquerque EX (1993) Diversity of nicotinic acetylcholine receptors in rat hippocampal neurons. I. Pharmacological and functional evidence for distinct structural subtypes. J Pharmacol Exp Ther 265:1455-1473.

Aosaki T, Tsubokawa H, Ishida A, Watanabe K, Graybiel AM, Kimura M (1994) Responses of tonically active neurons in the primate's striatum undergo systematic changes during behavioral sensorimotor conditioning. J Neurosci 14:3969-3984.

Aosaki T, Kimura M, Graybiel AM (1995) Temporal and spatial characteristics of tonically active neurons of the primate's striatum. J Neurophysiol 73:1234-1252.

Apicella P, Scarnati E, Schultz W (1991) Tonically discharging neurons of monkey striatum respond to preparatory and rewarding stimuli. Exp Brain Res 84:672-675.

Bolam JP, Clarke DJ, Smith AD, Somogyi P (1983) A type of aspiny neuron in the rat neostriatum accumulates $[3 \mathrm{H}] \mathrm{g}$-aminobutyric acid: combination of golgi-staining, autoradiography, and electron microscopy. J Comp Neurol 213:121-134.

Calabresi P, Centonze D, Gubellini P, Pisani A, Bernardi G (1998) Endogenous ACh enhances striatal NMDA-responses via M1-like muscarinic receptors and PKC activation. Eur J Neurosci 10:2887-2895.

Calabresi P, Centonze D, Gubellini P, Bernardi G (1999) Activation of M1-like muscarinic receptors is required for the induction of corticostriatal LTP. Neuropharmacology 38:323-326.

Calabresi P, Centonze D, Gubellini P, Pisani A, Bernardi G (2000) Acetylcholine-mediated modulation of striatal function. Trends Neurosci $23: 120-126$.

Chang HT, Kita H (1992) Interneurons in the rat striatum: relationships between parvalbumin neurons and cholinergic neurons. Brain Res 574:307-311.

Clements JD, Silver RA (2000) Unveiling synaptic plasticity: a new graphical and analytical approach. Trends Neurosci 23:105-113.

DeBoer P, Abercrombie ED (1996) Physiological release of striatal acetylcholine in vivo: modulation by D1 and D2 dopamine receptor subtypes. J Pharmacol Exp Ther 277:775-783.

Doods HN, Mathy MJ, Davidesko D, van Charldorp KJ, de Jonge A, van Zwieten PA (1987) Selectivity of muscarinic antagonists in radioligand and in vivo experiments for the putative M1, M2 and M3 receptors. J Pharmacol Exp Ther 242:257-262.

Frazier CJ, Rollins YD, Breese CR, Leonard S, Freedman R, Dunwiddie TV (1998) Acetylcholine activates an alpha-bungarotoxin-sensitive nicotinic current in rat hippocampal interneurons, but not pyramidal cells. J Neurosci 18:1187-1195.

Gerfen CR, Wilson CJ (1996) The basal ganglia. In: Handbook of chemical neuroanatomy (Swanson, LW, Bjorklund A, Hokfelt T, ed), pp 371-468. Amsterdam: Elsevier.

Guo JZ, Tredway TL, Chiappinelli VA (1998) Glutamate and GABA release are enhanced by different subtypes of presynaptic nicotinic receptors in the lateral geniculate nucleus. J Neurosci 18:1963-1969.

Hikosaka O, Sakamoto M, Usui S (1989) Functional properties of monkey caudate neurons. II. Visual and auditory responses. J Neurophysiol 61:799-813.

Horikawa K, Armstrong WE (1988) A versatile means of intracellular labeling: injection of biocytin and its detection with avidin conjugates. J Neurosci Methods 25:1-11.

Howe AR, Surmeier DJ (1995) Muscarinic receptors modulate N-, P-, and L-type $\mathrm{Ca}^{2+}$ currents in rat striatal neurons through parallel pathways. J Neurosci 15:458-469.

Jaeger D, Kita H, Wilson CJ (1994) Surround inhibition among projec- tion neurons is weak or nonexistent in the rat neostriatum. J Neurophysiol 72:1-4.

Kawaguchi Y (1993) Physiological, morphological, and histochemical characterization of three classes of interneurons in rat neostriatum. J Neurosci 13:4908-4923.

Kawaguchi Y, Wilson CJ, Augood SJ, Emson PC (1995) Striatal interneurones: chemical, physiological and morphological characterization. Trends Neurosci 18:527-535.

Kita H (1993) GABAergic circuits of the striatum. Prog Brain Res 99:51-72.

Kita H (1996) Glutamatergic and GABAergic postsynaptic responses of striatal spiny neurons to intrastriatal and cortical stimulation recorded in slice preparations. Neuroscience 70:925-940.

Kita H, Kitai ST (1988) Glutamate decarboxylase immunoreactive neurons in rat neostriatum: their morphological types and populations. Brain Res 447:346-352.

Kita H, Kosaka T, Heizmann CW (1990) Parvalbumin-immunoreactive neurons in the rat neostriatum: a light and electron microscopic study. Brain Res 536:1-15.

Koós T, Tepper JM (1999) Inhibitory control of neostriatal projection neurons by GABAergic interneurons. Nat Neurosci 2:467-472.

Kubota Y, Mikawa S, Kawaguchi Y (1993) Neostriatal GABAergic interneurones contain NOS, calretinin or parvalbumin. NeuroReport 5:205-208.

Limberger N, Spath L, Starke K (1986) A search for receptors modulating the release of gamma-[3H]aminobutyric acid in rabbit caudate nucleus slices. J Neurochem 46:1109-1117.

Marchi M, Sanguineti P, Raiteri M (1990) Muscarinic receptors mediate direct inhibition of GABA release from rat striatal nerve terminals. Neurosci Lett 116:347-351.

McGehee DS, Heath MJ, Gelber S, Devay P, Role LW (1995) Nicotine enhancement of fast excitatory synaptic transmission in CNS by presynaptic receptors. Science 269:1692-1696.

Plenz D, Kitai ST (1998) Up and down states in striatal medium spiny neurons simultaneously recorded with spontaneous activity in fastspiking interneurons studied in cortex-striatum-substantia nigra organotypic cultures. J Neurosci 18:266-283.

Radcliffe KA, Fisher JL, Gray R, Dani JA (1999) Nicotinic modulation of glutamate and GABA synaptic transmission of hippocampal neurons. Ann NY Acad Sci 868:591-610.

Schultz W, Apicella P, Romo R, Scarnati E (1995) Context-dependent activity in primate striatum reflecting past and future events. In: Models of information processing in the basal ganglia (Houk JC, Davis JL, Beiser DG, ed), pp 11-29. Cambridge, MA: A Bradford Book.

Smolders I, De Klippel N, Sarre S, Ebinger G, Michote Y (1995) Tonic GABAergic modulation of striatal dopamine release studied by in vivo microdialysis in the freely moving rat. Eur J Pharmacol 284:83-91.

Stricker C, Field AC, Redman SJ (1996) Statistical analysis of amplitude fluctuations in EPSCs evoked in rat CA1 pyramidal neurones in vitro. J Physiol (Lond) 490:419-441.

Stricker C, Redman S (1994) Statistical models of synaptic transmission evaluated using the expectation-maximization algorithm. Biophys $\mathrm{J}$ 67:656-670.

Sugita S, Uchimura N, Jiang ZG, North RA (1991) Distinct muscarinic receptors inhibit release of gamma-aminobutyric acid and excitatory amino acids in mammalian brain. Proc Natl Acad Sci USA 88:2608-2611.

Szabo B, Dorner L, Pfreundtner C, Norenberg W, Starke K (1998) Inhibition of GABAergic inhibitory postsynaptic currents by cannabinoids in rat corpus striatum. Neuroscience 85:395-403.

Timmerman W, Abercrombie ED (1996) Amphetamine-induced release of dendritic dopamine in substantia nigra pars reticulata: D1-mediated behavioral and electrophysiological effects. Synapse 23:280-291.

Zhou J, Shapiro MS, Hille B (1997) Speed of $\mathrm{Ca}^{2+}$ channel modulation by neurotransmitters in rat sympathetic neurons. J Neurophysiol 77:2040-2048.

Zoli M, Lena C, Picciotto MR, Changeux JP (1998) Identification of four classes of brain nicotinic receptors using beta2 mutant mice. J Neurosci 18:4461-4472. 